\title{
Cable tension estimation by means of vibration response and moving mass technique
}

\author{
Andrea Bellino, Stefano Marchesiello, Alessandro Fasana and Luigi Garibaldi ${ }^{\mathrm{a}}$
}

Dynamics \& Identification Research Group, Politecnico di Torino, Dipartimento di Meccanica, C.so Duca degli Abruzzi 24, 10129 Torino, Italia

Received 25 February 2010, Accepted 23 April 2010

\begin{abstract}
This paper approaches a novel technique to estimate cable tension simply based on its vibration response. The vibration response has been quite extensively adopted in the past due to its simplicity and, mainly, because the inverse approach allows the tension estimation with the cable in its original site. A first tentative approach consists in using a certain number of experimentally measured natural frequencies to be introduced in the theoretical vibration formula; this formula, however, involves also the cable length, the cable mass per unit length and its flexural rigidity. Unfortunately, some problems arise in its application to real structures, such as the case of suspended and cable-stayed bridges, because the exact cable length cannot be measured (it appears at the fourth exponent in the vibration formula); moreover section and weight can be estimated within a certain degree of accuracy, whilst the boundary conditions are often defined with difficulty. A novel extension of the method is here proposed, which takes advantage from a moving mass travelling on the cable. This is the case occurring when cables are verified with magnetic-based technology to detect rope faults and cross section reduction. In this way, the extracted natural frequencies are varying with time due to the moving load, and hence they have to be extracted adopting a time-varying approach. Although some approximation linked to the shape modification must be introduced, a simple iterative procedure can be settled, by considering the cable length as an unknown. An estimation of the equivalent length is given, and successively this value is used to obtain an estimation of the cable tension.
\end{abstract}

Key words: Cables / moving mass / equivalent length / tension estimation / time-varying identification

\section{Introduction}

During the last years, the interest towards the tensional study of cables has grown because of the construction of many cable-stayed bridges, now very popular because of their aesthetic appeal, structural efficiency and ease of construction.

At the moment, the major suspended bridge is the Akashi Kaikyo in Japan, with a span length of $1991 \mathrm{~m}$, but a longer span (about $3300 \mathrm{~m}$ ) is expected for the Messina Strait Bridge [1]. Another long bridge to be mentioned is the Tsing Ma Bridge in Hong Kong, with its $1337 \mathrm{~m}$ of span length [2].

It is well known that cable-stayed bridges are generally exposed to wind, rain, ice and long-term fatigue during their design life [3] and consequently the current tension cannot be supposed equal to the original tension.

The cable monitoring can be done in two ways: by making the passage of a magnetic-based inspection device, to detect rope faults and cross-section reduction,

\footnotetext{
${ }^{a}$ Corresponding author: luigi.garibaldi@polito.it
}

and by studying the tensional state of the cable. As a result, the cable tension is an important index for assessing overall structural health and condition of cable-supported bridges [4].

The tensional study is one of the most important issues in this engineering field. The current available techniques allow distinguishing two types of methods: the direct ones, which measure the tension by a hydraulic jack or a strain gauge, and the vibration methods, which calculate the tension from the estimated natural frequencies. The latter ones have received increasing attention because of their simplicity and speediness [5].

When analyzing long cables, with large diameters, the natural frequencies are not only influenced by the cable tension, but also by flexural rigidity, sag-span ratio and cable inclination $[1,6]$. Au et al. [7] studied the determination of natural frequencies and mode shapes of cablestayed bridges by the finite element method (FEM) and by the dynamic stiffness method (DSM).

The simplest vibrational method uses the relation proper of the taut string theory, but this procedure is 


\section{Nomenclature}

\begin{tabular}{|ll|}
\hline$A$ & cross-sectional area $\left(\mathrm{m}^{2}\right)$ \\
$d$ & sag $(\mathrm{m})$ \\
$f_{r}$ & natural frequency of the cable for the $r$-mode \\
$f_{r, m}$ & natural frequency of the cable for the $r$-mode, \\
& in presence of a mass on it \\
$E$ & Young's modulus $\left(\mathrm{N}^{-2}{ }^{-2}\right)$ \\
$I$ & moment of inertia $\left(\mathrm{m}^{4}\right)$ \\
$g$ & gravity acceleration $\left({\left.\mathrm{m} . \mathrm{s}^{-2}\right)}^{-2}\right)$ \\
$H(t)$ & dynamic tension $(\mathrm{N})$ \\
$L$ & horizontal tension $(\mathrm{N})$ \\
$L_{0}$ & length of the cable $(\mathrm{m})$ \\
$m$ & horizontal length $(\mathrm{m})$ \\
$s$ & vead mass $(\mathrm{kg})$ \\
$T$ & tension $(\mathrm{N})$ \\
$v$ & displacement in the $y$-direction $(\mathrm{m})$ \\
$V$ & load velocity $\left(\mathrm{m} . \mathrm{s}^{-1}\right)$ \\
$w$ & displacement in the $z$-direction $(\mathrm{m})$ \\
$y(x)$ & geometric shape of the cable \\
$x_{m}$ & load position $(\mathrm{m})$ \\
$\delta$ & sag-to-span ratio \\
$\mu$ & mass per unit length $\left(\mathrm{kg} \cdot \mathrm{m}{ }^{-1}\right)$ \\
$\vartheta$ & inclination angle of the cable $(\mathrm{rad})$ \\
\hline
\end{tabular}

strictly limited to flat long slender cables. If the flexural rigidity is taken into account, then the most used method is the linear regression, applied on a relation that links directly the natural frequencies to the tension and the bending stiffness of the cable [5]. Considering also the sag-extensibility, the most adopted approach is the use of practical formulas proposed by Zui et al. [6].

One of the problems arising with vibrational methods is the inapplicability to cable systems whose analytical solution is not known. Moreover, usually these types of methods need information about the cable properties, such as the length and the density per unit length. When analyzing real cables, it is very difficult to measure these cable properties with a high precision, both for technical problems and for the presence of not well defined boundary conditions. For this reason, the cable length will be renamed equivalent length, to highlight that this is not an absolute concept but it is referred to the assumed cable model.

The objective of this paper is to propose a new method for the estimation of the cable tension, but keeping the cable length as an unknown. To achieve this target, a moving load crossing the cable can be used, in order to have more independent equations to write. This new system is time-varying, i.e. with modal parameters changing instant by instant [8]. Moreover, this approach can be also applied when a mass is simply placed in a certain position along the cable.

Many studies were conducted about cables with attached masses, especially in the field of ocean engineering, because of the carrying of vessels, cameras and hydrophones. Sergev and Iwan [9] found the experimental

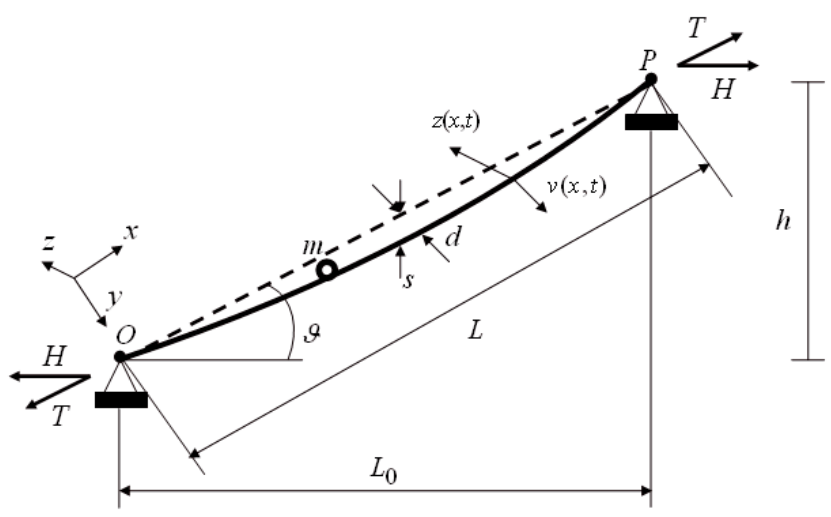

Fig. 1. The considered model of cable.

and theoretical natural frequencies and mode shapes of strings with attached masses, by considering the wave propagation equation. Cheng and Perkins [10] used the Hamilton's principle to derive the equations of motion of a sagged cable supporting a discrete mass. Qassab and Nair [11] studied the free vibration of catenary elastic cables with attached mass, using the wavelet-Galerkin method.

The present paper starts with an analytical description of an inclined simply supported cable, taking the flexural rigidity into account, but neglecting the sagextensibility, i.e. by neglecting the deviation from the straight line of its axis, due to the cable weight. Successively, the transit of the moving load is modelled as a concentrated mass moving with a constant velocity, and the time-varying natural frequencies are calculated. Then, the method for the estimation of the equivalent length and the cable tension is proposed. Eventually, a numerical example and an experimental example, realized in the laboratory of the Department of Structures at the Politecnico di Torino, are presented to show the reliability of the method.

\section{Model of cable}

Let us consider a simply supported cable with a moving load passing over it, as in Figure 1.

The left support $\mathrm{O}$ is the origin of the coordinates and the direction OP is taken as the $x$-coordinate.

The equations of motion of the cable without any moving load are given by [3]:

$$
\begin{gathered}
E I \frac{\partial^{4} v(x, t)}{\partial x^{4}}-T \frac{\partial^{2} v(x, t)}{\partial x^{2}}-h(t) \frac{\mathrm{d}^{2} y(x)}{\mathrm{d} x^{2}}+\mu \frac{\partial^{2} v(x, t)}{\partial t^{2}}=0 \\
E I \frac{\partial^{4} w(x, t)}{\partial x^{4}}-T \frac{\partial^{2} w(x, t)}{\partial x^{2}}+\mu \frac{\partial^{2} w(x, t)}{\partial t^{2}}=0
\end{gathered}
$$

where $E I$ is the cable bending rigidity, $v(x, t)$ and $w(x, t)$ represent the deflection in the $y$-direction and in the $z$ direction, $T$ is the static cable tension ( $H$ is its horizontal 
component), $h(t)$ is the dynamic tension, $y(x)$ is the geometric shape of the cable and $\mu$ is the mass per unit length of the cable.

Equation (1) is called equation of the in-plane motion, while Equation (2) is called equation of the out-of-plane motion.

Naming $d$ the cable sag, $s$ the vertical sag and $L_{0}$ the cable horizontal length, the sag-to-span ratio $\delta=s / L_{0}$ is supposed to be sufficiently small. The effect of $h(t)$ concerns only symmetric modes, in particular the first one; this is why it will be removed in the method. As a consequence of previous considerations, as also in [6], Equation (1) can be re-written as

$$
E I \frac{\partial^{4} v(x, t)}{\partial x^{4}}-T \frac{\partial^{2} v(x, t)}{\partial x^{2}}+\mu \frac{\partial^{2} v(x, t)}{\partial t^{2}}=0
$$

In view of the fact that Equations (2) and (3) have the same form, it is sufficient to analyze one of them, in particular that referring to the in-plane motion.

Imposing the variable separation $v(x, t)=W(x) q(t)$, the eigenproblem is formulated as follows

$$
\frac{\mathrm{d}^{4} W(x)}{\mathrm{d} x^{4}}-\frac{T}{E I} \frac{\mathrm{d}^{2} W(x)}{\mathrm{d} x^{2}}=\beta^{4} W(x)
$$

where $\beta^{4}=\frac{\omega^{2} \mu}{E I}$ and $q(t)=\sin (\omega t+\alpha)$.

After having imposed the boundary conditions for a simply supported cable, the $r$-natural frequency of the cable is found to be linked to the tension and to the flexural rigidity in the following manner (vibration formula):

$$
\left(\frac{f_{r}}{r}\right)^{2}=\frac{1}{4 \mu L^{2}} T+\frac{r^{2} \pi^{2}}{4 \mu L^{4}} E I
$$

\subsection{Presence of the moving load}

If moving loads are present, then equation (5) is no more valid. In the following analysis, the masses of the moving loads are considered quite small (but not negligible) with respect to the cable mass, as actually happens when using the cable inspection device.

The transit of the moving mass on a cable is a problem similar to the case of a moving mass on a beam, as done in a previous work [8]. In such a way, the equation of motion of the coupled cable-mass system is [12]:

$$
[M(t)]\{\ddot{q}(t)\}+[C(t)]\{\dot{q}(t)\}+[K(t)]\{q(t)\}=g\{\tau(t)\}
$$

where $g$ is the gravity acceleration and $\{q(t)\}$ the modal coordinates. The systems matrices for a single moving mass can be written as

$$
\begin{aligned}
& {[M(t)]=[I]_{n \times n}+m X(t)\{\Phi(\xi)\}\{\Phi(\xi)\}^{\mathrm{T}}} \\
& {[C(t)]=2 m V X(t)\{\Phi(\xi)\}\left\{\Phi^{I}(\xi)\right\}^{\mathrm{T}}} \\
& {[K(t)]=\left[\omega^{2}\right]+m V^{2} X(t)\{\Phi(\xi)\}\left\{\Phi^{I I}(\xi)\right\}^{\mathrm{T}}} \\
& \{\tau(t)\}=-m X(t)\{\Phi(\xi)\}
\end{aligned}
$$

where $m$ and $V$ are the mass and the velocity of the moving load, $\{\Phi(\xi)\}$ the mass-normalized mode shapes of the cable evaluated at the mass position $\xi=V t,\left[\omega^{2}\right]$ is the diagonal matrix containing the square of the first $n$ angular frequencies of the cable, $X(t)$ is a window function, defined by unit step functions $u$ in the following manner:

$$
X(t)=u(t)-u(t-l / V)
$$

The displacements can be found by using the mode superposition:

$$
w(x, t)=\sum_{i=1}^{n} \phi_{i}(x) q_{i}(t)
$$

Since the mass of the moving load is supposed small with respect to the cable mass, the terms out of the main diagonal of the mass matrix $[M(t)]$ can be ignored. Since real velocities cannot significantly alter the stiffness matrix, as it has been demonstrated by another research under development not reported here for brevity's sake, the effect of the load velocity is neglected. The natural frequencies of the cable subjected to the transit of a mass can be written as:

$$
f_{r, m}=\frac{f_{r}}{\sqrt{1+\frac{2 m}{\mu L} \sin \left(\frac{\pi r}{L} x_{m}\right)^{2}}}
$$

where $x_{m}$ indicates the position of the load along the cable. Equation (5) can be written as:

$$
\frac{f_{r, m}^{2}}{r^{2}}\left(1+\frac{2 m}{\mu L} \sin \left(\frac{\pi r}{L} x_{m}\right)^{2}\right)=\frac{1}{4 \mu L^{2}} T+\frac{\pi^{2} r^{2}}{4 \mu L^{4}} E I
$$

\section{Method of the equivalent length}

In this section the concept of equivalent length is firstly introduced, and then a method for the estimation of the cable tension is presented.

\subsection{The concept of equivalent length}

The relations expressed by Equations (5) and (14) work well if the cable properties, such as length and density per unit length, are known. To obtain a good estimation of the cable tension, the values of these characteristics must be as accurate as possible. When analyzing real cables, for example, it is very difficult to measure the length with a high precision, both for technical problems and because sometimes cables have not well defined boundary conditions. In Figure 2, an example of staycable bridge, with a magnification on the cable connection to the ground, is shown.

In the considered example, the cable is not simply linked to the ground but its low ending part is inserted in 




(a)

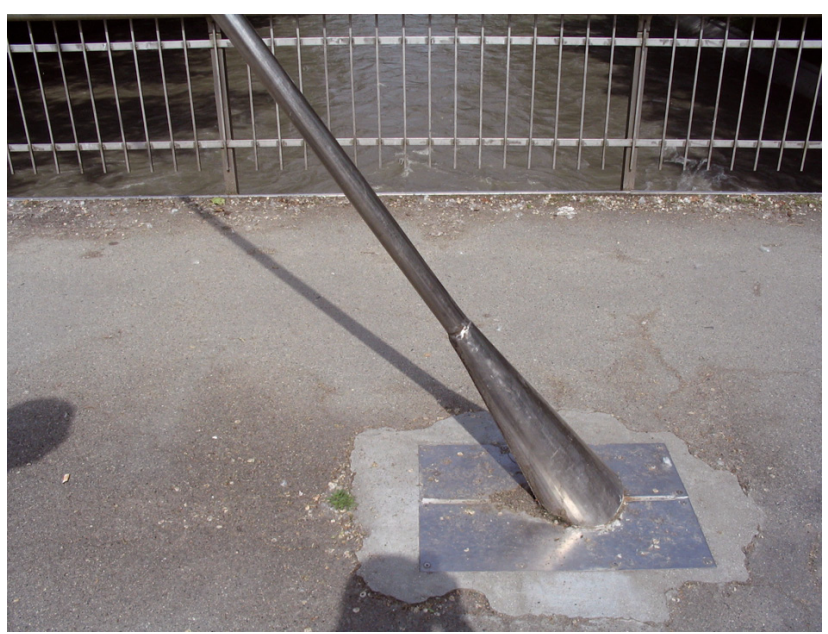

(b)

Fig. 2. Cable-stayed bridge (a) and a magnification on the metallic guide that links the cable to the ground (b).

a metallic guide, thus the choice of appropriate boundary conditions is difficult.

A possible solution to this problem may be to consider the cable length as an unknown, and to write an additional independent equation. The cable length that will be found is referred to as equivalent length, i.e. the length that corresponds to the natural frequencies extracted and to the known cable parameters.

\subsubsection{The procedure}

The objective of this section is to present three independent equations for the three unknowns of the cable: the length, the tension and the bending stiffness.
Starting from Equation (5), let us consider two different modes $p$ and $q$ and perform the difference between the first two members:

$$
\frac{f_{p}^{2}}{p^{2}}-\frac{f_{q}^{2}}{q^{2}}=\frac{\pi^{2}\left(p^{2}-q^{2}\right)}{4 \mu L^{4}} E I
$$

Since the contribution of the tension has been deleted, it is possible to write

$$
K_{1}^{p, q}=\frac{4 \mu}{\pi^{2}} \frac{\left(\frac{f_{p}}{p}\right)^{2}-\left(\frac{f_{q}}{q}\right)^{2}}{p^{2}-q^{2}}=\frac{E I}{L^{4}}
$$

Starting newly from Equation (5), another relation can be formulated by eliminating the contribution due to flexural rigidity:

$$
K_{2}^{p, q}=\frac{4 \mu\left(\frac{1}{p^{2}}\left(\frac{f_{p}}{p}\right)^{2}-\frac{1}{q^{2}}\left(\frac{f_{q}}{q}\right)^{2}\right)}{\frac{1}{p^{2}}-\frac{1}{q^{2}}}=\frac{T}{L^{2}}
$$

Better estimations of the values $K_{1}^{p, q}$ and $K_{2}^{p, q}$ are given by averaging the results coming from all the available natural frequencies. We rename these values as $K_{1}$ and $K_{2}$.

A third equation, independent from the first two, is needed. It can come only from the case with the moving load crossing the cable. Considering Equation (14) for the mode $p$, with the mass in a certain position $x_{m}$,

$$
\begin{gathered}
\frac{f_{p, m}^{2}}{p^{2}}\left(1+\frac{2 m}{\mu L} \sin \left(\frac{\pi p}{L} x_{m}\right)^{2}\right)=\frac{1}{4 \mu L^{2}} T+\frac{\pi^{2} p^{2}}{4 \mu L^{4}} E I \\
4 \mu \frac{f_{p, m}^{2}}{p^{2}}\left(1+\frac{2 m}{\mu L} \sin \left(\frac{\pi p}{L} x_{m}\right)^{2}\right)=K_{2}+\pi^{2} p^{2} K_{1} \\
1+\frac{2 m}{\mu L} \sin \left(\frac{\pi p}{L} x_{m}\right)^{2}=\frac{K_{2}+\pi^{2} p^{2} K_{1}}{4 \mu \frac{f_{p, m}^{2}}{p^{2}}}
\end{gathered}
$$

Imposing

$$
g_{x_{m}}^{p}(Z)=1+\frac{2 m}{\mu Z} \sin \left(\frac{\pi p}{Z} x_{m}\right)^{2}-\frac{K_{2}+\pi^{2} p^{2} K_{1}}{4 \mu \frac{f_{p, m}^{2}}{p^{2}}}
$$

then the final step is the search of the zero of $g_{x_{m}}^{p}(Z)$, which will give the equivalent length. A better estimation of it is given by the mean of all the values coming from different modes and different load positions.

Once having obtained the equivalent length, the tension and the flexural rigidity can be obtained by inverting Equations (16) and (17) or by using the linear regression on Equation (5).

The entire procedure can be also applied if other parameters are unknown, as for example the mass per unit length. In this case, $K_{1}$ and $K_{2}$ must also contain the unknown $\mu$, and two relations of the type of Equation (19) must be written.

The mass has been introduced in order to help us to write additional equations. In practice, therefore, a known mass can be placed on a certain position along the cable under examination, to obtain an estimation of the tension, if some cable characteristics, as the length, are not exactly known. 
Table 1. Cable properties.

\begin{tabular}{cc}
\hline Cable property & Value \\
\hline Length & $L=29.939 \mathrm{~m}$ \\
Section & $S=0.0013 \mathrm{~m}^{2}$ \\
Mass per unit length & $\mu=10.2661 \mathrm{~kg} . \mathrm{m}^{-1}$ \\
Tension & $T=268880 \mathrm{~N}$ \\
Flexural rigidity & $E I=23522 \mathrm{~N} . \mathrm{m}^{2}$ \\
\hline
\end{tabular}

\section{Numerical application}

In this section, a numerical example consisting of the tensional study of a horizontal simply supported cable is performed. The characteristics of the cable, shown in Table 1, are those of a real cable of a stay-cable bridge.

The time histories are generated by integrating Equation (6), with $3 \%$ of Gaussian noise added to the accelerations. The time intervals have been taken equal to the time crossings of the loads, which travel always at $V=1 \mathrm{~m} \cdot \mathrm{s}^{-1}$, and the sampling frequency is $120 \mathrm{~Hz}$. Two masses have been chosen for the time-varying analysis, respectively equal to $5 \mathrm{~kg}$ and $10 \mathrm{~kg}$.

The output-only identification is performed both with the classical stochastic subspace identification [13], for the time-invariant systems, and with the ST-SSI (Short Time Stochastic Subspace Identification) method [8], for the time-varying systems. The latter is based on the frozen technique, consisting of the classical LTI identification, but performed in different windows, in which the whole time period is divided.

Since the low modes are affected by sag-extensibility, during the analysis only the modes after the fourth have been taken into account.

After the time-varying identification, five different positions of the moving masses have been considered for the estimation of the cable tension.

In Table 2, the results obtained by the application of the method for both the masses are presented. The linear regression method has been used to extract the tension and the flexural rigidity.

As expected, the case with the larger mass produces more relevant errors. Moreover, the estimation of the cable tension is more accurate with respect to the estimation of the flexural rigidity.

\section{Experimental application}

The experimental application was conducted in the Laboratory of the Department of Structures, at the Politecnico di Torino. The system considered is a steel rope (see Fig. 3) for hoisting applications: tower cranes, elevators, non-guides loads.

The rope is composed by 114 threads, according to the section shown in Figure 4. In Table 3, all the rope characteristics are listed.

The tension has been measured by means of calibrated masses.

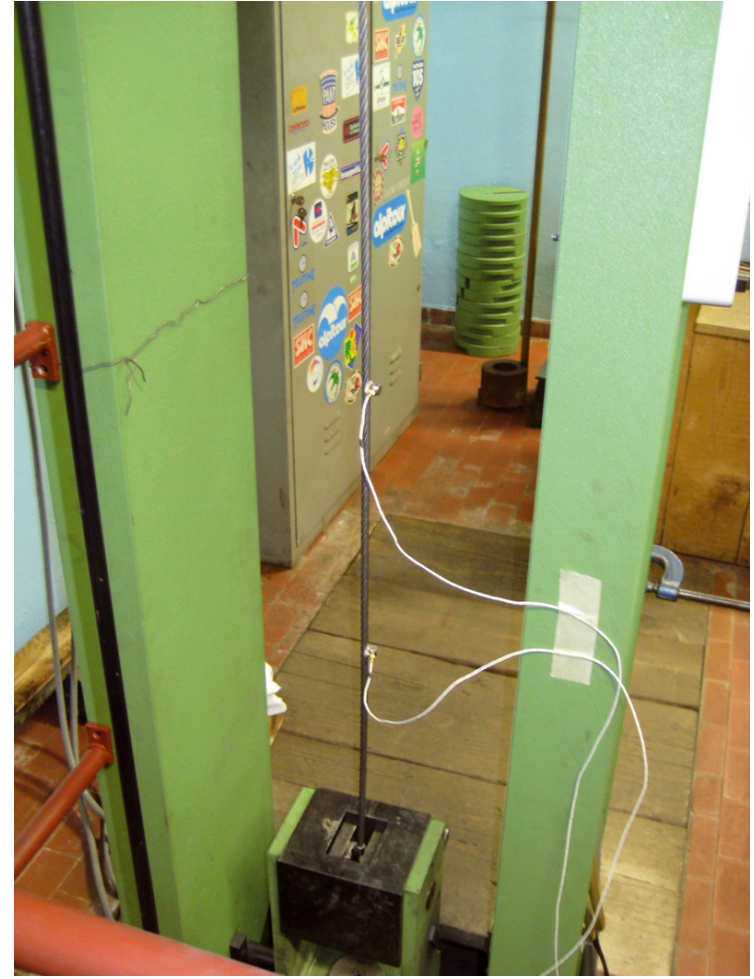

Fig. 3. The experimental system considered.

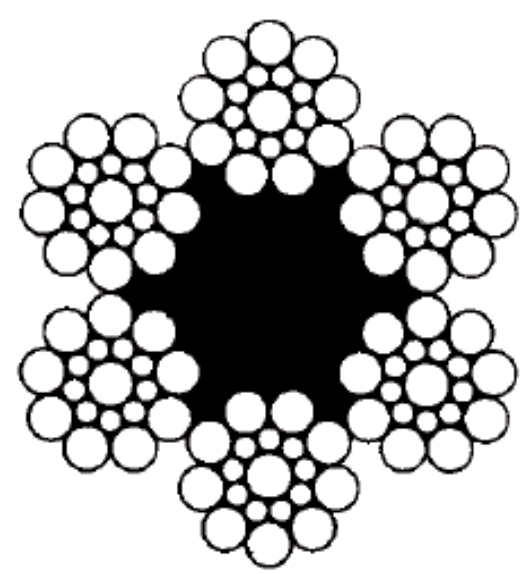

Fig. 4. The section of the rope considered.

In order to estimate the rope tension, only a single mass has been added on it, at a fixed distance of $0.55 \mathrm{~m}$ from the low attachment of the rope.

Since the masses of the two accelerometers placed along the rope are not irrelevant in this case, it is important to eliminate this contribution by modifying the extracted frequencies:

$$
f_{p, \mathrm{~N}}=f_{p, \mathrm{~A}} \sqrt{1+\frac{2 m_{\mathrm{a}}}{\mu L}\left(\sin \left(\frac{\pi p}{L} x_{\mathrm{a} 1}\right)^{2}+\sin \left(\frac{\pi p}{L} x_{\mathrm{a} 2}\right)^{2}\right)}
$$


Table 2. Results obtained by the method, with $3 \%$ of noise.

\begin{tabular}{llll}
\hline$m=5 \mathrm{~kg}$ & $L_{\mathrm{eq}}=29.776 \mathrm{~m}$ & $T=2.658 \times 10^{5} \mathrm{~N}$ & $E I=2.308 \times 10^{4} \mathrm{~N} . \mathrm{m}^{2}$ \\
& $e r_{L}=0.545 \%$ & $e r_{T}=1.141 \%$ & $e r_{E I}=1.883 \%$ \\
\hline$m=10 \mathrm{~kg}$ & $L_{\mathrm{eq}}=29.618 \mathrm{~m}$ & $T=2.630 \times 10^{5} \mathrm{~N}$ & $E I=2.259 \times 10^{4} \mathrm{~N} . \mathrm{m}^{2}$ \\
& $e r_{L}=1.072 \%$ & $e r_{T}=2.186 \%$ & $e r_{E I}=3.947 \%$ \\
\hline
\end{tabular}

Table 3. Rope properties.

\begin{tabular}{cc}
\hline Rope property & Value \\
\hline Type & $6 \times(9+9+1)$ SEALE \\
Length & $L=1.58 \mathrm{~m}$ \\
Diameter & $d=9.00 \times 10^{-3} \mathrm{~m}$ \\
Diameter of big threads & $d_{1}=0.70 \times 10^{-3} \mathrm{~m}$ \\
Diameter of small threads & $d_{2}=0.35 \times 10^{-3} \mathrm{~m}$ \\
Section & $S=63.59 \times 10^{-6} \mathrm{~m}^{2}$ \\
Mass per unit length & $\mu=0.2982 \mathrm{~kg} \cdot \mathrm{m}^{-1}$ \\
Applied tension & $T=16480.8 \mathrm{~N}$ \\
\hline
\end{tabular}

where $f_{p, \mathrm{~N}}$ indicates the rope frequencies without any mass, $f_{p, \mathrm{~A}}$ are the frequencies of the configuration given by the rope and the accelerometers, $m_{\mathrm{a}}=3.25 \times 10^{-3} \mathrm{~kg}$ is the mass of each accelerometer, $x_{\mathrm{a} 1}=0.331 \mathrm{~m}$ and $x_{\mathrm{a} 1}=0.760 \mathrm{~m}$ are the sensor positions.

Three types of acquisitions have been executed, as listed in Table 4. The sampling frequency has been always taken equal to $6400 \mathrm{~Hz}$.

The identification method is no more the ST-SSI because there are no moving loads, therefore only the classical stochastic subspace identification is applied.

Looking at the small mass acquisition, the differences between two consecutive natural frequencies of $f_{p, \mathrm{~A}}$ and $f_{p, \mathrm{~N}}$ are shown respectively in Figures $5 \mathrm{a}$ and $5 \mathrm{~b}$.

It is easy to see that, in the first case, the natural frequencies do not have a clear trend, while in the second case they assume the disposition proper of a cable, whose flexural rigidity affects the modes increasingly [5].

In Figure 6 the estimation of the rope equivalent length is shown for all the modes and for both the cases with an added mass.

In this analysis, only the modes after the third have been considered. This is the reason why in Figure 6 the equivalent length is equal to zero for the first modes. Moreover, in case (a) for the sixth mode it was not possible to find a zero of the function $g_{x_{m}}^{p}$.

The final results obtained by the proposed method are presented in Table 5.

The most interesting aspect is the link between the equivalent length and the tension. In the second case the equivalent length found is very near to the measured one, but the tension is quite different. Contrarily, in the first case the equivalent length is very small but the tension is very close to the measured one. Since the rope is not simply supported at the ends, it is not possible to say if the estimation of the equivalent length is good or not. Consequently, the unique parameter that must be taken

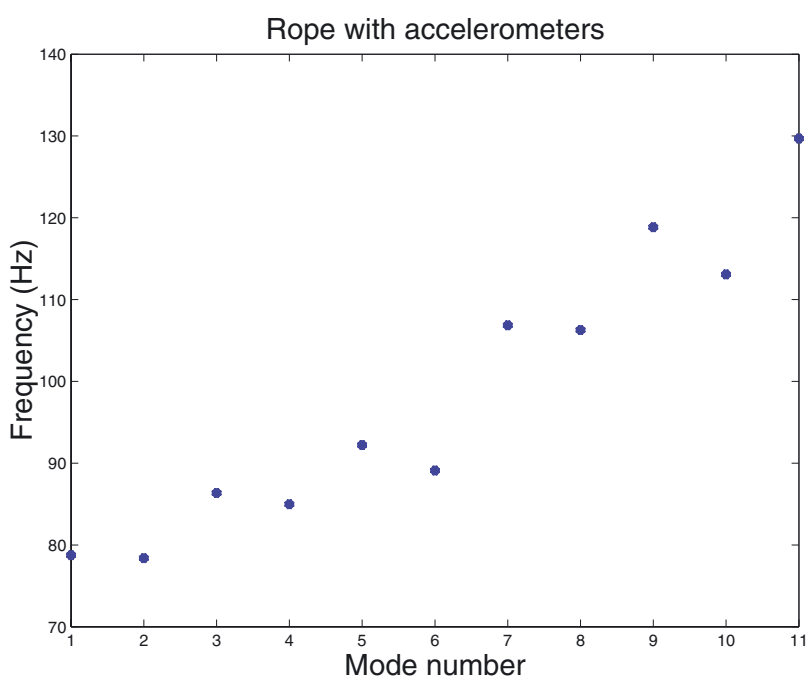

(a)

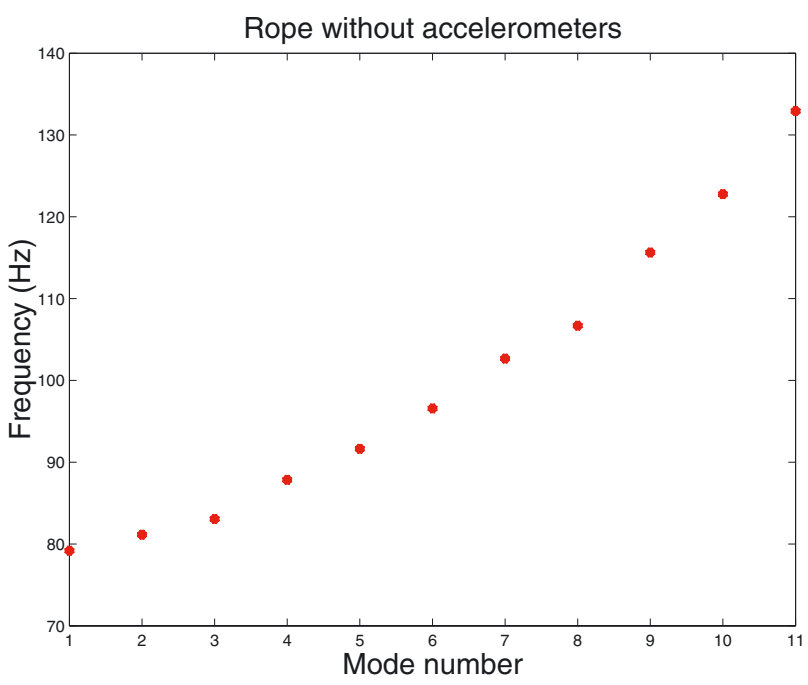

(b)

Fig. 5. Difference between two consecutive natural frequencies for the rope with accelerometers (a) and eliminating the effect of the accelerometers (b).

into account to validate the method is the estimation of the rope tension.

\section{Conclusions}

In this article, a new method for the estimation of the cable tension is proposed. It is based on the concept of 
Table 4. Characteristics of the different acquisitions.

\begin{tabular}{cccc}
\hline Denomination & Mass position & Added mass & \% of the rope mass \\
\hline 0 & - & - & - \\
1 & $x_{m}=0.55 \mathrm{~m}$ & $m=19.6 \times 10^{-3} \mathrm{~kg}$ & $4.16 \%$ \\
2 & $x_{m}=0.55 \mathrm{~m}$ & $m=60.0 \times 10^{-3} \mathrm{~kg}$ & $12.73 \%$ \\
\hline
\end{tabular}

Table 5. Results obtained by the method.

\begin{tabular}{ccccc}
\hline Test & $\begin{array}{c}\text { Eq. length } \\
(\mathrm{m})\end{array}$ & $\begin{array}{c}\text { Tension } \\
(\mathrm{N})\end{array}$ & $\begin{array}{c}\text { Tension error } \\
(\%)\end{array}$ & $\begin{array}{c}\text { Flexural rigidity } \\
\left(\mathrm{N} . \mathrm{m}^{2}\right)\end{array}$ \\
\hline 1 & 1.5035 & $1.6452 \times 10^{4}$ & 0.18 & 15.0286 \\
2 & 1.5649 & $1.7825 \times 10^{4}$ & 8.16 & 17.6420 \\
\hline
\end{tabular}

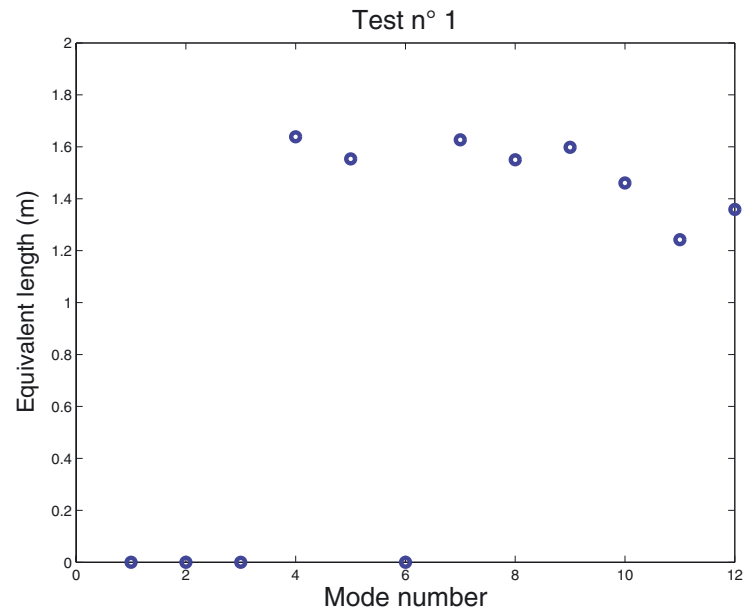

(a)

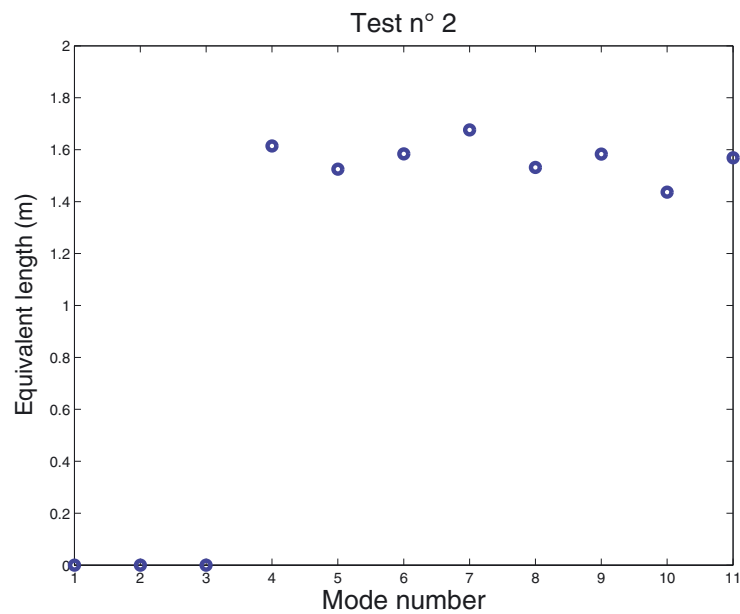

(b)

Fig. 6. Estimation of the equivalent length by using the smaller mass (a) and by using the larger mass (b).

equivalent length, i.e. the length corresponding to the natural frequencies extracted and to the known cable characteristics.
The relationships used derive from the simply supported cable model, the unique case in which a simple connection between all the parameters (tension, flexural rigidity, mass per unit length, length, natural frequencies) is possible to find. This is not a limitation but it is the central point of the method: the relationships are simple and the equivalent length is useful to include in this analysis other boundary configurations.

The proposed method is reliable both with the addition of a single mass in a certain position (shown in the experimental case) and with the transit of a mass over the cable (shown in the numerical case). An important aspect is the choice of the added mass: it must be not too large, in order to calculate the natural frequencies by using only the diagonal terms of the mass and stiffness matrix. Otherwise, the method results cannot be considered reliable.

Acknowledgements. Authors wish to thank laboratories of the DISTR - Structural and Geotechnical Engineering Department of the Politecnico di Torino, for the invaluable helping in the realization of the experimental test. A special thanks to the colleagues Marco Alessio, Maria Pavano and Pietro Provenzano.

\section{References}

[1] G. Ricciardi, F. Saitta, A continuous vibration analysis model for cables with sag and bending stiffness, Eng. Struct. 30 (2008) 1459-1472

[2] Y.L. Xu, J.M. Ko, Z. Yu, Modal analysis of tower-cable system of Tsing Ma long suspension bridge, Eng. Struct. 19 (1997) 857-867

[3] N. Bouaanani, Numerical investigation of the modal sensitivity of suspended cables with localized damage, J. Sound Vib. 292 (2006) 1015-1030

[4] Y.Q. Ni, J.M. Ko, G. Zheng, Dynamic analysis of largediameter sagged cables taking into account flexural rigidity, J. Sound Vib. 257 (2002) 301-319

[5] B.H. Kim, T. Park, Estimation of cable tension force using the frequency-based system identification method, J. Sound Vib. 304 (2007) 660-676 
[6] H. Zui, T. Shinke, Y. Namita, Practical formulas for estimation of cable tension by vibration method, Am. Soc. Civil Eng. J. Struct. Eng. 122 (1996) 651-656

[7] F.T.K. Au, Y.S. Cheng, Y.K. Cheung, D.Y. Zheng, On the determination of natural frequencies and mode shapes of cable-stayed bridges, Appl. Math. Mod. 25 (2001) 1099-1115

[8] A. Bellino, G. Garibaldi, S. Marchesiello, Time-varying output-only identification of a cracked beam, Key Engineering Materials 413-414 (2009) 643-650

[9] S.S. Sergev, W.D. Iwan, The natural frequencies and mode shapes of cables with attached masses, J. Energy Resour. Technol. 103 (1981) 237-242
[10] S.P. Cheng, N.C. Perkins, Closed-form vibration analysis of sagged cable/mass suspensions, J. Appl. Mech. 59 (1992) 923-928

[11] M. Al-Qassab, S. Nair, Wavelet-Galerkin method for the free vibrations of an elastic cable carrying an attached mass, J. Sound Vib. 270 (2004) 191-206

[12] B. Biondi, G. Muscolino, New improved series expansion for solving the moving oscillator problem, J. Sound Vib. 281 (2005) 99-117

[13] P. Van Overschee, B. De Moor, Subspace Identification for Linear Systems: Theory, Implementation, Applications, Kluwer Academic Publishers, Boston London Dordrecht, 1996 